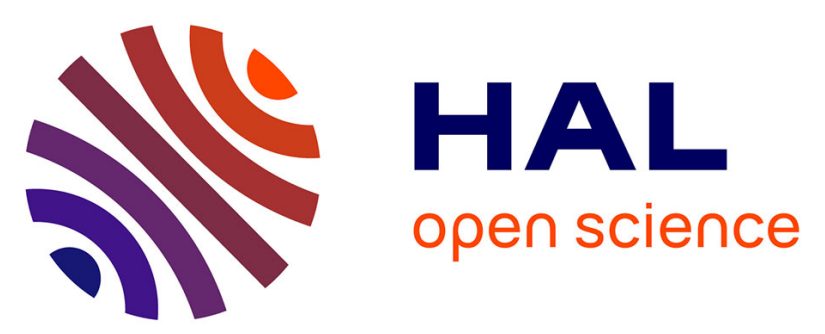

\title{
Les inspections primaires de l'éducation nationale: dotations, pratiques et effets sur le fonctionnement des écoles et les acquis des élèves
}

\author{
Jean-Pierre Jarousse, Christine Leroy-Audouin, Alain Mingat
}

\section{To cite this version:}

Jean-Pierre Jarousse, Christine Leroy-Audouin, Alain Mingat. Les inspections primaires de l'éducation nationale: dotations, pratiques et effets sur le fonctionnement des écoles et les acquis des élèves. 1995, pp.4. hal-03206408

\section{HAL Id: hal-03206408 \\ https://hal.science/hal-03206408}

Submitted on 10 Jun 2021

HAL is a multi-disciplinary open access archive for the deposit and dissemination of scientific research documents, whether they are published or not. The documents may come from teaching and research institutions in France or abroad, or from public or private research centers.
L'archive ouverte pluridisciplinaire HAL, est destinée au dépôt et à la diffusion de documents scientifiques de niveau recherche, publiés ou non, émanant des établissements d'enseignement et de recherche français ou étrangers, des laboratoires publics ou privés. 


\title{
Les Inspections primaires de l'Education Nationale Dotations, pratiques et effets sur le fonctionnement des écoles et les acquis des élèves
}

\author{
J.P. Jarousse, C. Leroy-Audouin et A. Mingat *
}

* Nous remercions Jean Ferrier pour sa contribution à ce travail.

Les Inspections de l'Education Nationale (IEN) ont été créées au milieu du 19 ème siècle avec comme but premier de contrôler le fonctionnement des écoles ; la dimension idéologique et républicaine était très présente, le rattachement initial des inspecteurs étant auprès des Préfets. Progressivement, les dimensions d'encadrement pédagogique et d'équipe de circonscription ont pris corps et il apparaît clairement que les inspecteurs primaires ont joué un rôle très important, d'une part en tant que corps intermédiaire pour mettre en oeuvre les dispositions prises au niveau national et d'autre part, en tant que corps d'appui organisationnel et pédagogique pour le fonctionnement des écoles au niveau local.

Depuis la fin des années soixante, la dimension de contrôle et d'inspection des personnels s'est progressivement atténuée pour donner à l'inspecteur et à son équipe des fonctions davantage tournées vers l'animation et la formation. Le rôle des inspecteurs s'est par ailleurs sans doute élargi avec les développements de la déconcentration de l'administration, les Zones d'Education Prioritaires et les projets d'école. En 1995, on compte environ 1200 circonscriptions sur l'ensemble du territoire national.

D'une façon générale, les activités effectives des inspecteurs et des équipes de circonscription sont relativement peu connues. Une enquête conduite par la Direction de l'Evaluation et de la Prospective et la Direction des Ecoles au cours de l'année 1989-90, dans l'Académie de Lille, permet d'apporter quelques éclairages sur ce que sont ces inspections et leurs équipes, les moyens qu'elles mobilisent et les activités qu'elles mettent en oeuvre (des informations sur 27 inspections ont été collectées). Par ailleurs, les données recueillies permettent également d'explorer dans quelle mesure ces actions influencent le fonctionnement du système éducatif d'une part dans la régulation des différenciations inter-écoles et d'autre part dans les modes d'organisation interne de chacune d'entre elles et in fine, les acquisitions des élèves.

\section{De fortes variations dans les moyens alloués aux inspections}

* Un premier fait à souligner est la forte variabilité des caractéristiques des circonscriptions, que l'on considère leur degré d'urbanisation ou le nombre de leurs maîtres. Celui-ci est en moyenne de 247, avec une dispersion notable; mises à part quatre circonscriptions dont le nombre de maitres est inférieur à 200, les 23 circonscriptions restantes ont des tailles qui s'échelonnent de façon régulière entre 200 et 356 maîtres. Au-delà de ces aspects quantitatifs, les circonscriptions sont également très différentes sur le plan de leurs populations scolaires, qu'on les caractérise par la distribution des origines sociales (la proportion d'enfants de cadres peut varier de 1 à 5) ou la nationalité des élèves (la proportion des étrangers varie de 3 à plus de 25\%).

* Les circonscriptions se distinguent par ailleurs selon la mobilité de leurs enseignants (le taux de demande de mutation varie, en 1989, de 8 à 35\%) et selon leur taux d'absentéisme moyen; le rapport des demi-journées d'absence des maîtres au total annuel de demi-journées de classe varie en effet de 0,2 à 10,4 pour-cent. Sur ce dernier point, on observe une tendance à un moindre absentéisme dans les zones rurales ainsi que, paradoxalement, dans les zones plus populaires.

* Concernant les dotations, les moyens à disposition des circonscriptions sont les affectations de personnels et l'équipement de leur secrétariat. Sur ces deux plans, on observe des différences notables d'un lieu à un autre. 
. si toutes les circonscriptions sont dotées d'une secrétaire à temps plein, le nombre de personnes permanentes (conseillers pédagogiques, Secrétaire de Circonscription Préélémentaire et Elémentaire, animateur informatique en équivalent temps plein) est en moyenne de 3,85 (y compris l'IEN), avec une distribution très continue entre 2,5 et 5,5. L'équipe peut bénéficier en outre de la participation de

conseillers pédagogiques par discipline (le cas le plus fréquent correspondant à la présence d'un conseiller supplémentaire). Il est à noter que l'on n'observe pas de relation entre le nombre de ces personnels supplémentaires et celui de l'équipe de circonscription de "base".

- l'équipement matériel du secrétariat de la circonscription apparait assez conventionnel, voire rudimentaire, eu égard à l'état et à la diffusion de la technologie : seules $44 \%$ des inspections disposent d'une photocopieuse, un micro-ordinateur n'est présent que dans moins d'une circonscription sur cinq. Dans une perspective plus pédagogique, deux-tiers des circonscriptions déclarent être équipées d'une bibliothèque pédagogique auprès de laquelle les enseignants peuvent se documenter.

* Tant sur le plan des personnels que des moyens matériels, la variété est grande d'une inspection à l'autre; l'analyse montre qu'il n'y a pas substitution entre ces deux éléments (pas plus de matériel quand il y a moins de personnel) mais complémentarité (d'autant plus de moyens matériels que le personnel est plus nombreux, comme s'il s'agissait d'une "consommation administrative"). La variabilité observée à la fois dans les caractéristiques des circonscriptions (nombre de maitres, urbain/rural, composition sociale de la population) et dans les moyens (personnels et matériels) qu'elles mobilisent invite à rechercher dans quelle mesure l'une répond à l'autre, en examinant si la variabilité dans les moyens est déterminée par l'intensité des besoins à satisfaire et par les particularités des conditions locales.

. quand on met en regard le nombre des personnels de l'équipe de circonscription (y compris l'inspecteur) et celui des enseignants qui y sont en activité, le taux d'encadrement moyen est de 1 pour 46,7 maîtres; il est très variable d'une circonscription à l'autre, dans une fourchette de 1 pour 33 dans la circonscription apparemment la mieux dotée (circ. chargée de l'enseignement spécialisé non comprise) à 1 pour 108 dans celle qui l'est le moins (on observe une distribution relativement continue sur cet intervalle) : on peut donc trouver un nombre comparable de personnels dans une circonscription comptant 120 ou 320 maîtres. .il apparaît en outre que les dotations en personnels ne s'expliquent ni par la tonalité sociale de la circonscription, ni par son caractère plus ou moins rural : il n'y a pas de dotations supérieures en personnels dans les circonscriptions socialement difficiles et/ou géographiquement étendues. Quant aux dotations en matériels, on observe qu'elles ne dépendent ni du nombre de maîtres de la circonscription, ni de sa tonalité sociale; en revanche, les circonscriptions rurales apparaissent moins dotées. Au total, c'est donc globalement l'aléa qui domine les allocations de moyens aux inspections par l'administration.

\section{Des pratiques très variées d'une inspection à l'autre}

Sur la base du questionnaire, les inspecteurs déclarent adopter des pratiques différenciées dans leur exercice professionnel. Au-delà de la variété dans des conduites ponctuelles et isolées, les pratiques peuvent se lire dans des profils articulant les dimensions suivantes :

. une première distinction oppose les inspecteurs qui privilégient la dimension pédagogique de leur action (principalement menée en direction des enseignants et qui concerne de façon privilégiée les animations pédagogiques et la formation continue par exemple) à ceux pour lesquels la perspective gestionnaire prime (soit les activités liées à la gestion administrative, humaine et matérielle de la circonscription); ces deux groupes sont comparables en nombre.

. une seconde distinction oppose les inspecteurs dont les activités sont centrées sur les écoles et leur fonctionnement et ceux dont les activités manifestent un degré plus fort d'ouverture sur l'extérieur (relations avec les collectivités locales, missions...); sans qu'il y ait de recouvrement avec la dimension précédente (on trouve par exemple autant de pédagogues que de gestionnaires parmi ceux qui ont des activités principalement centrées sur les écoles), les effectifs des IEN "centrés" et "ouverts" sont approximativement équivalents.

. une troisième distinction concerne l'"intensité de l'activité" de l'inspection (mesurée par le nombre de formations, d'animations pédagogiques, de visites d'inspection) et oppose des inspecteurs qui sont relativement peu actifs à d'autres dont le degré de mobilisation est très élevé.

. une quatrième dimension manifeste le niveau d'intentionnalité des actions entreprises (formations, conférences pédagogiques, critères d'inspection) en 
référence aux apprentissages des élèves et aux résultats pédagogiques effectivement obtenus.

- enfin, une cinquième dimension concerne le style relationnel, tant avec les personnels de l'inspection qu'avec les directeurs d'école ou les maitres; elle s'exprime par un degré plus ou moins grand de directivité ou de convivialité de l'inspecteur dans ses relations de travail.

Sur le plan statistique, l'articulation de ces cinq dimensions, qui sont dans une assez large mesure indépendantes, permet de lire assez bien la variété des comportements des inspecteurs dans la gestion de leur circonscription, variété qui n'est que très modérément liée aux moyens disponibles dans l'inspection. Il apparaît donc tout à fait inadéquat de parler "génériquement" des inspecteurs ou des inspections comme s'il s'agissait d'une institution homogène, car c'est l'hétérogénéité des pratiques qui domine le paysage.

\section{Quelles pratiques se révèlent laisser des traces sur les écoles et les élèves?}

La description des pratiques des IEN montre combien est grande la variété d'une circonscription à l'autre; cela dit, pour se prononcer sur la pertinence à agir "de telle façon plutôt que telle autre" et éviter l'utilisation d'arguments normatifs douteux, il convient de disposer de références pour jauger la valeur de ces pratiques. Dans le système scolaire en général, les acquisitions des élèves constituent une balise importante, sachant que pour ce qui concerne les corps d'inspection en particulier, la capacité à homogénéiser le fonctionnement des écoles de la circonscription est aussi un indicateur pertinent.

* Les acquisitions des élèves ont elles-mêmes des dimensions multiples et il serait bien sûr préférable de disposer de mesures dans ces différentes dimensions; cela est évidemment très exigeant. De façon plus limitée, nous avons utilisé les acquis en français et en mathématiques à l'entrée au CE2 (épreuves nationales disponibles pour tous les enfants des 27 circonscriptions retenues). L'analyse de ces données révèle à la fois l'influence des caractéristiques individuelles des élèves et du contexte social des scolarisations; elle révèle aussi l'existence d'une forte composante locale (école ou classe) manifestant que des élèves de caractéristiques comparables réalisent des acquis scolaires sensiblement différents selon le lieu où ils sont scolarisés.
Une analyse plus spécifique montre qu'environ 12 pourcent (un huitième) de ces différences de lieu peuvent se lire au niveau de la circonscription; d'une circonscription à l'autre, des élèves de caractéristiques semblables tendent bien, en moyenne, à avoir des acquisitions différentes. Alors que l'échelle des acquis scolaires a un écart-type de 15 points, on observe des effets de circonscriptions dont l'intensité est de plus ou moins 4,5 points; ces écarts, s'ils ne sont pas très importants, sont tout de même tout à fait notables.

Ces "effets de circonscription" peuvent alors être rapprochés des pratiques observées dans la gestion de l'inspection. On observe que les écarts précédemment identifiés s'expliquent en partie par les pratiques développées. Le fait que l'IEN ait des activités ouvertes sur l'extérieur ou plutôt centrées sur l'école ainsi qu'une orientation plutôt gestionnaire ou pédagogique se révèle sans effet sur ce que les élèves de la circonscription apprennent; en revanche, une pratique relationnelle plus directive et un activisme intense de l'IEN semblent plutôt défavorables : l'activité est probablement utile mais il n'est sans doute pas nécessaire qu'elle prenne des dimensions spectaculaires; la qualité relationnelle apparaît comme un élément plus déterminant que la quantité des activités déployées.

Une dimension des pratiques des inspecteurs se révèle associée de façon plus significative aux acquisitions des élèves. Il s'agit du degré relatif d'attention portée au fonctionnement de l'école en lui-même d'une part, et aux résultats des élèves d'autre part. Ainsi certains inspecteurs signalent plus que d'autres dans leurs pratiques (critères d'inspection, visée des animations pédagogiques,..) une orientation plus spécifique sur ce que les élèves apprennent. Les résultats obtenus montrent que c'est dans ces circonscriptions que les acquis scolaires des élèves (après contrôle de leurs caractéristiques personnelles) sont effectivement plus élevés.

* En ce qui concerne l'homogénéisation de l'efficacité pédagogique des écoles/classes d'une même circonscription, on observe une forte variété d'une inspection à l'autre. Celle-ci va au-delà des différences de public entre les écoles car les écarts enregistrés ici sont nets de la composition sociale du public des différentes écoles de la circonscription. Cela dit, on peut distinguer deux origines dans ces écarts : une qui renvoie à des facteurs généraux ou contextuels qui rendent plus ou moins difficiles la gestion de la circonscription et le maintien d'un degré faible d'hétérogénéité; une autre qui tient en elle-même à l'action des inspecteurs. Sur le premier aspect, il apparaît 
que l'homogénéité est plus difficile à atteindre dans les circonscriptions les plus rurales; par ailleurs, on observe qu'une équipe de circonscription plus nombreuse ne constitue pas un facteur favorable à l'homogénéisation des résultats des écoles. Concernant le second aspect, les caractéristiques personnelles de l'IEN, de même que ses pratiques de gestion de la circonscription, n'ont globalement que peu d'incidence; seule l'intensité de la formation continue se révèle à cet égard positive. Au total, il subsiste de fortes différences d'une inspection à l'autre dans le degré d'homogénéité des pratiques pédagogiques de leurs écoles.

\section{Une régulation d'ensemble assez insatisfaisante du système éducatif primaire}

* Les résultats obtenus peuvent sans doute nourrir des réflexions pour i) l'amélioration des modalités d'affectation des moyens aux différentes circonscriptions et ii) la régulation des actions des inspecteurs et la formation initiale/continue de ceux-ci. Cela dit, au-delà du constat concernant les moyens des inspections et les pratiques des inspecteurs, les résultats invitent à des interrogations plus fondamentales pour la politique éducative et la gestion du système éducatif primaire.

* On a en effet des indices forts du caractère inadéquat de l'action régulatrice des Inspecteurs auprès des enseignants, en observant de façon directe les pratiques dans les classes et les résultats des élèves. Il existe, sur le plan des pratiques des enseignants, une variabilité très forte; ainsi, le temps consacré à la lecture en Cours Préparatoire varie de 7 à 15,5 heures par semaine (Mingat, Suchaut, 1995, Cahier de l'Irédu n56) et des chiffres d'amplitude équivalente sont dégagés en classe de CE2 (Altet et al., 1994, Dossiers Education et Formations n ${ }^{\circ} 44$ ). En termes de résultats des élèves, les observations faites dans cette recherche (convergentes avec celles d'autres travaux) dégagent l'existence de substantielles différences d'efficacité pédagogique d'une classe à une autre, et montrent que cette variabilité inter-classe n'est finalement que faiblement régulée par l'appartenance à une même inspection, comme le rappelle le graphique ci-contre mettant en regard le degré d'hétérogénéité inter-écoles dans la cir- conscription et son efficacité pédagogique moyenne.

* Les inspecteurs primaires ont joué dans le passé un rôle très important pour assurer au système éducatif un niveau minimum d'homogénéité nationale; il n'est pas sûr qu'ils remplissent aujourd'hui ce rôle de façon aussi satisfaisante. La variété des pratiques des inspecteurs est sans doute un indice de l'incertitude dans laquelle ils se trouvent quant à leur fonction. Les nombreux changements intervenus au cours des 25 dernières années dans leur formation et leurs relations institutionnelles sont, de façon plus fondamentale, l'indice de la faible détermination de l'administration quant au rôle qu'elle souhaite leur faire jouer. Le système éducatif évolue et, si les modes de fonctionnement des inspecteurs primaires des années 1880-1960 ne sont évidemment plus adaptés, il est probable qu'on n'a pas encore trouvé une formule satisfaisante aux conditions actuelles de l'enseignement.

Graphique : Relation entre le degré d'hétérogénéité de la circonscription et son efficacité pédagogique moyenne

Indisponible dans la version Acrobat

Au total, et sur la base de ces éléments empiriques, ces résultats manifestent, d'une part que l'idée d'un système éducatif primaire homogène est, dans de très larges mesures, mise à mal, et d'autre part, que l'influence du corps d'inspection primaire dans ce domaine est au mieux, faible.

\section{Pour en savoir plus :}

FERRIER (Jean). "Les inspecteurs des écoles primaires". Thèse Sciences de l'éducation. Dijon, juin 1995. 1010 p.

JAROUSSE (Jen-Pierre), LEROY-AUDOUIN (Christine), MINGAT (Alain). "Les inspections de l'Education Nationale au niveau primaire : disparités, dotations, pratiques et effets sur le fonctionnement des écoles et les acquis des élèves". Revue Française de Pédagogie. A paraître (34 p.)

Rappel des dernières Notes (contre $5 \mathrm{~F}$ en timbres ou un coupon réponse international)

95/4 CAP et BEP : Bourgogne : une insertion typée selon la spécialité

95/6 Les groupements d'élèves dans l'école primaire rurale en France : efficacité pédagogique et intégration des élèves au collège

"Les Notes de l'irédu" est une collection à parution irrégulière

Chaque numéro peut être obtenu contre $5 \mathrm{~F}$ en timbres auprès du service documentation de l'irédu I.S.S.N. en cours 